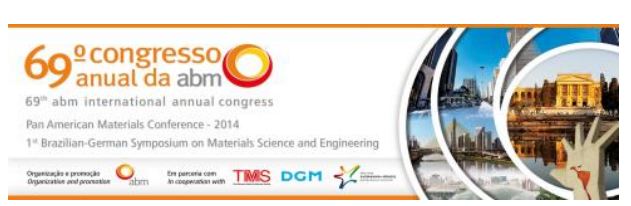

Tema: Iniciação Científico-tecnológica

\title{
AVALIAÇÃO DA INFLUÊNCIA DE DIFERENTES GEOMETRIAS DE FERRAMENTAS DE TREFILAÇÃO POR MEIO DE SIMULAÇÃO COMPUTACIONAL*
}

\author{
Eduardo Rocha Costa ${ }^{1}$ \\ Juliana Zottis² \\ Alexandre da Silva Rocha ${ }^{3}$
}

\section{Resumo}

Barras trefiladas podem não apresentar o perfil desejado pelos seus fabricantes após o processo de produção, por isso, este trabalho tem por objetivo avaliar diferentes geometrias de ferramentas, mais precisamente, o ângulo de entrada e região de calibração $(\mathrm{Hc})$ da mesma. Para modelamento numérico do processo, foi utilizado o software Deform ${ }^{\mathrm{TM}}$, que possibilitou este estudo, com simulações da influência da ferramenta no perfil de tensões residuais dos produtos (barras de aço AISI1045). Foram analisados os diferentes comportamentos do material para processos com fieiras de ângulos de $15^{\circ}$ e $20^{\circ}$, com foco nas variações de tensões residuais visando investigar o produto final da trefilação. Em uma das principais comparações entre as fieiras anteriormente mencionadas, observou-se uma diferença de tensões residuais de aproximadamente $206 \mathrm{MPa}$ no centro das barras trefiladas, resultado que somado a distribuições assimétricas de tensões em relação ao ponto central de determinado corte da barra, podem ocasionar o seu empenamento.

Palavras-chave: Simulação computacional; Trefilação; Fieira.

\section{ANALYSIS OF THE INFLUENCE OF THE DRAWING DIE GEOMETRY BY MEANS OF COMPUTER SIMULATION}

\section{Abstract}

Drawn bars may not present the required profile for their manufacturers after its production process. For this reason this paper has as objective to study different die geometries, more precisely the drawing angle and bearing zone $(\mathrm{Hc})$ of the tool. For the numeric model process was used the Deform ${ }^{\mathrm{TM}}$ software which has enabled this study with die influence simulations for the products residual stress (AISI1045 still bars). Were analyzed the different material behavior in process with die angle of 15 and 20 degrees. The focus is on variations of residual stresses and to investigate the changes in the final product. In one of the main comparison between the drawing dies before said, was observed a difference of residual stress with nearly 206MPa in the drawing bar center, result which added with asymmetric stress distributions, in relation to the determined bar central point, may cause its bowing.

Keywords: Numerical simulation; Cold-drawing; Wire drawing die.

1 Graduando, Design de Produto, bolsista de iniciação científica LdTM, UFRGS, Porto Alegre, RS, Brasil; eduardo_costa2006@hotmail.com.

2 Mestre em Engenharia, Doutoranda LdTM, UFRGS, Porto Alegre, RS, Brasil; juliana.zottis@ufrgs.br.

3 Doutor em Engenharia, Prof. Dr. Pesquisador, LdTM, UFRGS, Porto Alegre, RS, Brasil; alexandre.rocha@ufrgs.br.

* Contribuição técnica ao 69 Congresso Anual da ABM - Internacional e ao 14ํㅡㄹ ENEMET - Encontro Nacional de Estudantes de Engenharia Metalúrgica, de Materiais e de Minas, 21 a 25 de julho de 2014, São Paulo, SP, Brasil. 


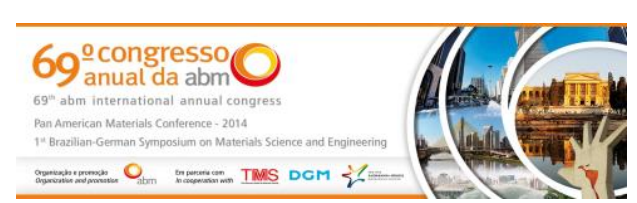

\section{INTRODUÇÃO}

A trefilação é um importante processo de conformação mecânica para fabricação de peças com simetria axial; atualmente uma boa alternativa para obter barras, fios e arames de diferentes diâmetros com boa precisão dimensional e menor custo de materiais e energia quando comparada com outros processos como laminação e extrusão $[1,2]$. Os produtos obtidos são geralmente utilizados para a fabricação de eixos de automóveis, máquinas industriais, ferramentas e máquinas têxteis [3]. Neste processo, o material tem sua seção transversal reduzida e seu comprimento aumentado, pois é tracionado através de uma ferramenta cônica, denominada fieira ou trefila $[4,5]$. Esta matriz cônica pode ser fabricada em aço ou carboneto de tungstênio e sua geometria consiste em quatro regiões ou zonas: região de entrada,que facilita a lubrificação para a passagem da barra, com ângulo maior do que o ângulo de trefilação; região de trefilação, com ângulo $(2 \alpha)$; região cilíndrica $(\mathrm{Hc})$, necessária por razões de fabricação e de manutenção da matriz; e finalmente a região de saída (ao contrário das outras) facilitando a saída do material $[6,2]$ A Figura 1 representa a matriz em corte, para que possa ser melhor visualizada.

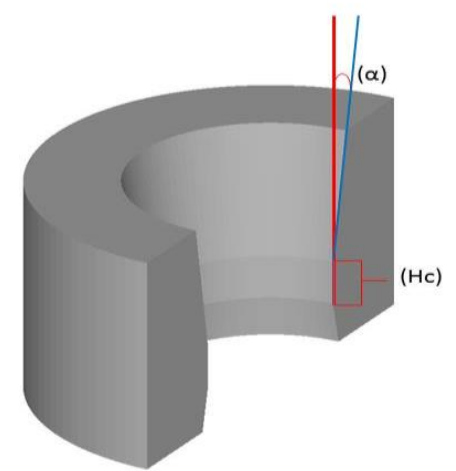

Figura 1. Desenho da fieira com representação do ângulo de trefilação $(\alpha)$ e região de calibração $(H c)$

A escolha dos parâmetros apropriados para a realização do processo de trefilação pode ser complexa quando se deseja controlar as características do produto final.

As principais variáveis envolvidas são o semi-ângulo $(\alpha)$ da matriz, a redução da barra $(r)$ e o coeficiente de atrito $\mu$, que, variando conforme a geometria das barras, estão relacionadas à interação entre barra e fieira [7].

Desde o século passado, teve-se um importante avanço nos estudos sobre a trefilação devido às exigências por melhorias de qualidade e produtividade. Mesmo assim, ainda não é possível eliminar satisfatoriamente as tensões residuais produzidas durante a conformação do material, que não sofre uma deformação plástica completamente homogênea em toda sua seção [5]. O perfil indesejável dessas tensões pode tanto modificar o comportamento mecânico e a resistência mecânica do material cilíndrico, quanto provocar trincas e distorções [5-8].

O desenvolvimento tecnológico dos processos de conformação mecânica, incluindo a trefilação, e de novos produtos têm sido visível nas indústrias, com a utilização de simulações numéricas pelo método de elementos finitos [3]. As vantagens do método consistem na capacidade de verificar soluções detalhadas das propriedades mecânicas em peças conformadas, em retirar dados de força, deformação, tensões, temperaturas, velocidades ou até distribuição de contatos de pressão [7].

Por fim, com a utilização da simulação numérica do processo, o objetivo deste trabalho consistiu na análise da influência dos principais parâmetros relacionados a

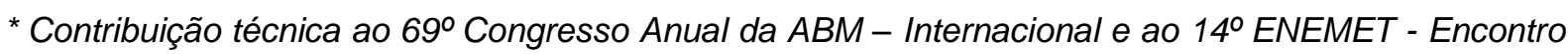
Nacional de Estudantes de Engenharia Metalúrgica, de Materiais e de Minas, 21 a 25 de julho de 2014, São Paulo, SP, Brasil. 


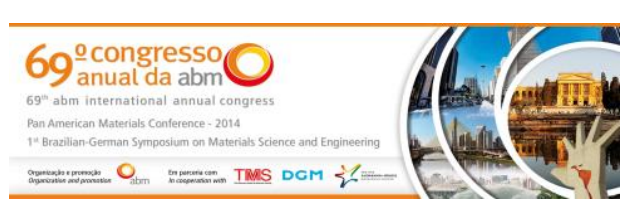

geometria da matriz: o semi-ângulo ( $\alpha$ ), na zona de entrada da fieira, e a região de calibração (Hc). Os outros parâmetros utilizados foram obtidos por um processo anterior com barras de aço AISI 1045.

\section{MATERIAIS E MÉTODOS}

O método dos elementos finitos (FEM) foi utilizado para realizar a análise da influência dos parâmetros da fieira no perfil das tensões residuais de barras de aço trefiladas. Para entender melhor a influência do ângulo da fieira e da região de calibração foram realizadas simulações com 5 diferentes ângulos, sendo $15^{\circ}$ e $20^{\circ}$ os principais, utilizando a modelagem tridimensional (3D) do software Deform ${ }^{\mathrm{TM}}$; assim como para o estudo da região de calibração $(\mathrm{Hc})$, com o modo 2D.

Para todas as simulações com ângulos diferentes foram usados os mesmos parâmetros, o atrito e a curva de escoamento da barra foram utilizados do estudo realizado por Zottis [7], sendo que a redução foi de aproximadamente $11 \%$. As propriedades do material da barra utilizados e os parâmetros para as simulações podem ser vistos, respectivamente nas Tabelas 1 e 2.

Tabela 1. Propriedades do aço AISI 1045

\begin{tabular}{|c|c|}
\hline Propriedades & Ângulos \\
\hline Material & $\begin{array}{l}\text { AISI } 1045 \\
{[70 C(20 C)]}\end{array}$ \\
\hline Módulo de Young & $210 \mathrm{GPa}$ \\
\hline $\begin{array}{ll}\text { Coeficiente } & \text { de } \\
\text { Poisson } & \\
\end{array}$ & 0,3 \\
\hline Limite de escoamento & $368,9 \mathrm{MPa}$ \\
\hline Curva de escoamento & $K_{f}=1292,8 \cdot \varphi^{0,2018}$ \\
\hline Temperatura & $20^{\circ} \mathrm{C}$ \\
\hline
\end{tabular}

Tabela 2. Parâmetros do processo.

\begin{tabular}{ll}
\hline Parâmetros Descrição & \\
\hline Diâmetro final & $20,250 \mathrm{~mm}$ \\
\hline Coeficiente de atrito Coulomb $\mu$ & 0,1 \\
\hline Velocidade do processo & $1250 \mathrm{~mm} / \mathrm{s}$ \\
\hline Tipo de função de escoamento & Von mises \\
\hline Regra de endurecimento & Isotrópico \\
\hline Método & Lagrangial \\
& Incremental \\
\hline Solver & Sparse \\
\hline Método de interação & Newton- \\
& Raphson \\
\hline
\end{tabular}

* Contribuição técnica ao 69 Congresso Anual da ABM - Internacional e ao 14ํㅡㄹ ENEMET - Encontro Nacional de Estudantes de Engenharia Metalúrgica, de Materiais e de Minas, 21 a 25 de julho de 2014, São Paulo, SP, Brasil. 

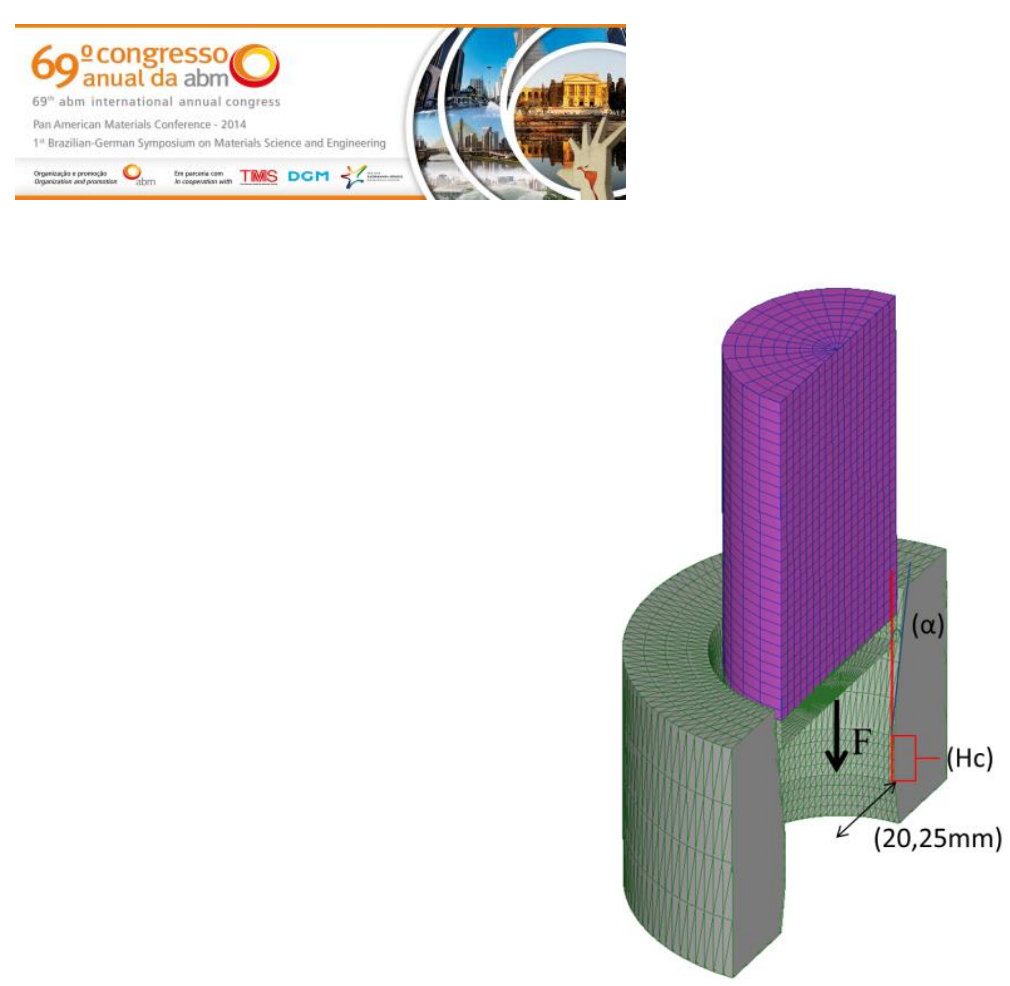

Figura 2. Corte de perfil do modelo base para as simulações.

A malha escolhida para a modelagem das barras foi a hexaédrica cujo modelo pode ser visto na Figura 2, pois é muito regular e tem seus elementos bem distribuídos em relação ao eixo $z$, podendo ser avaliada de diferentes formas, incluindo um acompanhamento dos pontos durante todas as etapas da simulação. Neste caso a malha foi produzida com 8 elementos no raio por aliar melhor tempo computacional e resultados satisfatórios em termos de tensão residual. A ferramenta em análise foi considerada rígida e as barras foram desenhadas em 2D e depois rotacionadas para serem utilizadas em 3D, sendo consideradas elasto-plásticas. Como o desenho para gerar os sólidos foi o mesmo pode-se assegurar, portanto, que os modelos foram desenvolvidos pelo mesmo método.

Realizaram-se simulações utilizando os ângulos entre 15 e $20^{\circ}\left(2 \alpha=17^{\circ} 30^{\prime}\right)$, sendo considerados ângulos teóricos ou ideais. Além destes ângulos teóricos, foi obtido também um ângulo de aproximadamente $14^{\circ} 22^{\prime}$, medida encontrada em uma fieira realmente utilizada na indústria. Portanto, uma simulação com o mesmo ângulo também fez parte da pesquisa.

Por fim, aplicando a equação do ângulo ótimo, que expressa a relação do atrito e redução para obter o semi-ângulo em que o processo teria menor gasto de energia. Através da Equação 1, o resultado obtido foi de aproximadamente $2 \alpha=10^{\circ}$.

$$
\alpha_{\text {otimo }}=\sqrt{\frac{3}{2} \cdot m \cdot \ln \left(\frac{D_{i}}{D_{f}}\right)}
$$

Equação 1

Para a análise da região de calibração foram variados os passes de 1 até 5 passes no modo 2D do software com os mesmos parâmetros utilizados para a variação dos ângulos. Foi mantido o ângulo de (2a) em $15^{\circ}$ para todas as trocas dos parâmetros. Tanto na análise dos diferentes ângulos quanto dos passes foram obtidos os valores das forças de trefilação e das tensões residuais pelo software de maneira semelhante.

Para as simulações em 3D e 2D, os valores de força podem ser mapeados da mesma forma. Os resultados das tensões são obtidos pelo mapeamento de pontos no centro das barras, em que no caso da simulação 2D será apenas de metade da seção, pois estas simulações foram feitas com utilização de um eixo de simetria. Os

\footnotetext{
* Contribuição técnica ao 69ำ Congresso Anual da ABM - Internacional e ao 14ํㅡㄹ ENEMET - Encontro Nacional de Estudantes de Engenharia Metalúrgica, de Materiais e de Minas, 21 a 25 de julho de 2014, São Paulo, SP, Brasil.
} 


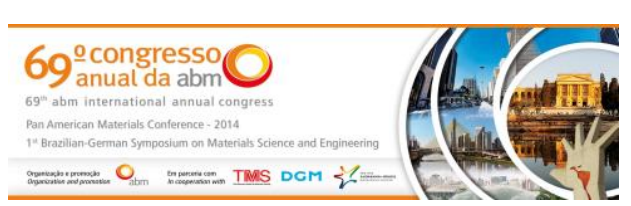

valores das tensões residuais são dados em cinco direções, mas as para este caso retira-se de três principais (axial, radial e tangencial). A Figura 3 mostra como foram feitas as situações 2D, para avaliar os passes.

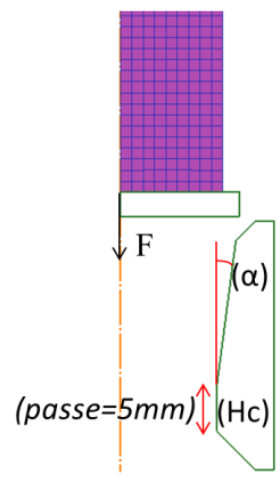

Figura 3. Modelo de simulação para avaliar os passes.

\section{RESULTADOS E DISCUSSÃO}

\subsection{Validação Numérica}

Os resultados das tensões foram avaliados no perfil axial radial e tangencial para melhor entendimento da influência dos ângulos e da região de calibração, assim puderam ser vistas as influências nos três perfis.

Outra resposta importante do software são os resultados de força para comparar o gasto de energia gasta no processo [9].

Tabela 3. Forças de trefilação retiradas no

\begin{tabular}{ll}
\multicolumn{2}{c}{ software. } \\
\hline Ângulo $\alpha(\stackrel{\circ}{)})$ & Força $(\mathrm{kN})$ \\
\hline 10,692 & 79,30 \\
\hline 14,358 & 74,29 \\
\hline 15 & 73,45 \\
\hline 17,5 & 78,42 \\
\hline 20 & 77,54 \\
\hline
\end{tabular}

Tabela 4. Forças de trefilação retiradas no software

\begin{tabular}{lc}
\hline Passes & Força $(\mathrm{kN})$ \\
\hline 1 & 72,18 \\
\hline 2 & 73,61 \\
\hline 3 & 76,34 \\
\hline 4 & 79,15 \\
\hline 5 & 81,94 \\
\hline
\end{tabular}

Avaliando as forças de trefilação (Tabelas 3 e 4) resultantes de cada parâmetro avaliado, observa-se uma elevação da força com o aumento do número de passes, conforme esperado. Por outro lado, quando se analisa a força em função do ângulo de trefilação, não é possível identificar uma tendência, sendo que a menor força encontrada foi de aproximadamente $73 \mathrm{kN}$, corresponde à trefilação de ângulo de trabalho a igual a $15^{\circ}$. Nota-se também que os ângulos de $10,692^{\circ} ; 17,5^{\circ}$ e $2^{\circ}$ 은 apresentaram valores de força em torno de $80 \mathrm{kN}$, variando, no máximo, em aproximadamente $3 \%$. Nota-se também que a simulação do ângulo ótimo não apresenta a menor força entre as demais, o que mostra uma diferença nos cálculos do software em relação ao que deveria ser a influência do ângulo ótimo.

Estas simulações foram todas feitas a partir de um modelo de referência, em que os parâmetros podem ser repetidos em um processo real e verificados com a literatura, tornando os experimentos deste trabalho válidos.

\footnotetext{
* Contribuição técnica ao 69ำ Congresso Anual da ABM - Internacional e ao 14ํㅡㄹ ENEMET - Encontro Nacional de Estudantes de Engenharia Metalúrgica, de Materiais e de Minas, 21 a 25 de julho de 2014, São Paulo, SP, Brasil.
} 


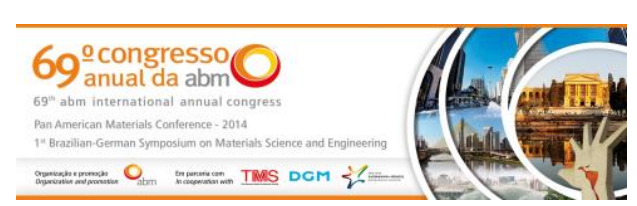

\subsection{Análise dos Ângulos da Ferramenta}

O Perfil de tensões residuais está relacionado aos ângulos de trefilação.

Os ângulos foram avaliados em 3D, com a retirada pontos no sentido horizontal no centro das barras trefiladas e obtidos os perfis das tensões após o processo.

A Figura 4 apresenta as seções de cortes dos perfis de tensão residual axial da barra após a simulação de trefilação, em que os pontos estão na direção horizontal (eixo $x$ ), e outra seção do perfil mostrando a distribuição das tensões ao longo do centro da barra.
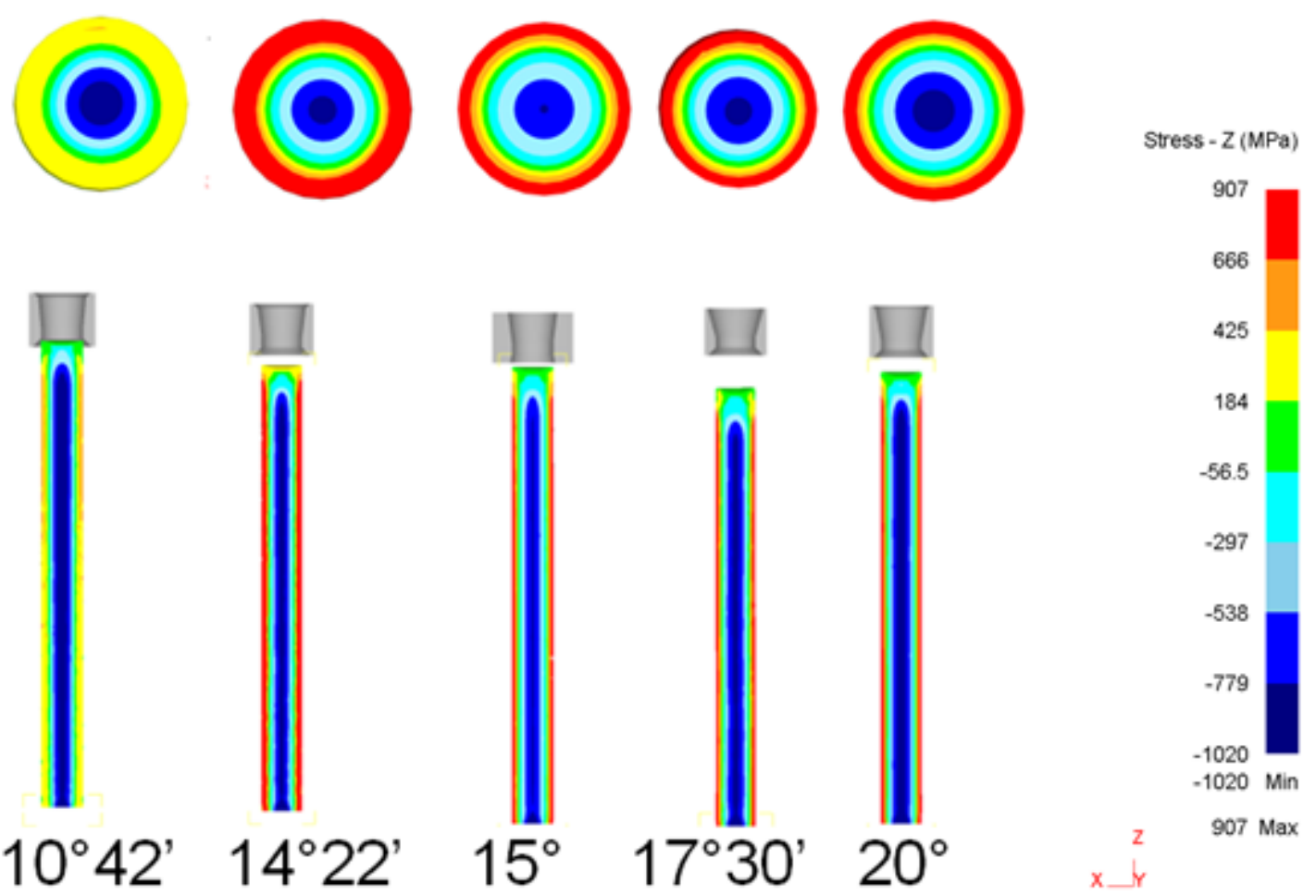

O valor do ângulo $2 \alpha$ está abaixo do correspondente perfil das tensões

Figura 4. Seções do centro das barras e cortes dos perfis e seus respectivos ângulos de trefilação abaixo.

As Tabelas 5, 6 e 7 mostram as diferenças encontradas no centro e na superfície das barras trefiladas.

Tabela 5. Tensões na direção Axial [MPa]

\begin{tabular}{lrrr}
\hline Angulo & Superfície & Centro & Superfície \\
\hline 10,692 & 406 & -970 & 356 \\
\hline 14,358 & 658 & -906 & 508 \\
\hline 15 & 729 & -925 & 682 \\
\hline 17,5 & 793 & -819 & 614 \\
\hline 20 & 756 & -892 & 721 \\
\hline
\end{tabular}

* Contribuição técnica ao 69ำ Congresso Anual da ABM - Internacional e ao 14ํㅡㄹ ENEMET - Encontro Nacional de Estudantes de Engenharia Metalúrgica, de Materiais e de Minas, 21 a 25 de julho de 2014, São Paulo, SP, Brasil. 


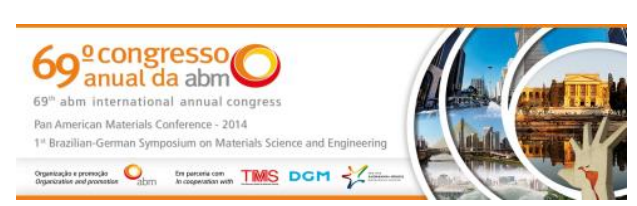

Tabela 6. Tensões na direção radial [MPa]

\begin{tabular}{lrrr}
\hline Angulo & Superfície & Centro & Superfície \\
\hline 10,692 & 316 & -357 & 372 \\
\hline 14,358 & 443 & -319 & 382 \\
\hline 15 & 478 & -326 & 441 \\
\hline 17,5 & 474 & -225 & 414 \\
\hline 20 & 463 & -284 & 424 \\
\hline
\end{tabular}

Tabela 7. Tensões na direção tangencial [MPa]

\begin{tabular}{lrrr}
\hline Angulo & Superfície & Centro & Superfície \\
\hline 10,692 & -51 & -371 & -30 \\
\hline 14,358 & -29 & -319 & -33 \\
\hline 15 & -23 & -327 & -30 \\
\hline 17,5 & -22 & -215 & 2 \\
\hline 20 & -21 & -285 & -26 \\
\hline
\end{tabular}

Pode-se notar no gráfico (perfil axial, Figura 5) que a fieira de $15^{\circ}$ apresentou maior tensão no centro e na superfície comparada com a fieira de $20^{\circ}$. Para a fieira com $17^{\circ} 30^{\prime}$, entre as duas principais, a curva apresenta redução das tensões negativas no centro (perfil axial) em relação às de 15 e $20^{\circ}$ se mantendo com os valores entre as outras duas.

A ferramenta real (com $2 \alpha=14^{\circ} 22^{\prime}$ ) apresentou uma redução das tensões do centro e aumento nas superficiais de aproximadamente $-19 \mathrm{MPa}$ e $100 \mathrm{MPa}$, respectivamente, em relação a de $15^{\circ}$. Já a fieira com ângulo ótimo teve o menor valor das tensões nas superfícies e a maior no centro em relação a todas.

As curvas podem ser visualizadas nos gráficos na Figura 5.

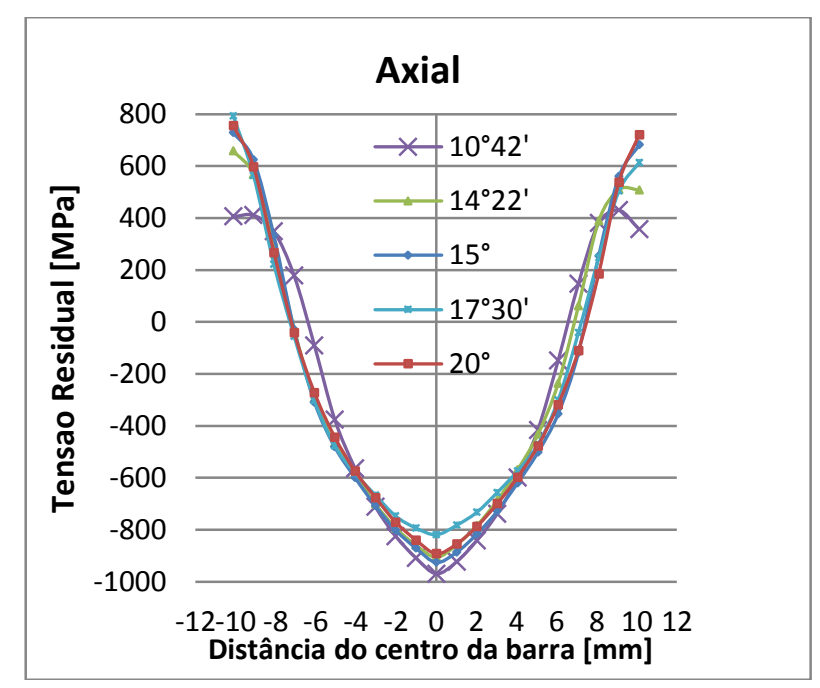

\footnotetext{
* Contribuição técnica ao $69^{\circ}$ Congresso Anual da ABM - Internacional e ao 14ํㅡㄹ ENEMET - Encontro Nacional de Estudantes de Engenharia Metalúrgica, de Materiais e de Minas, 21 a 25 de julho de 2014, São Paulo, SP, Brasil.
} 


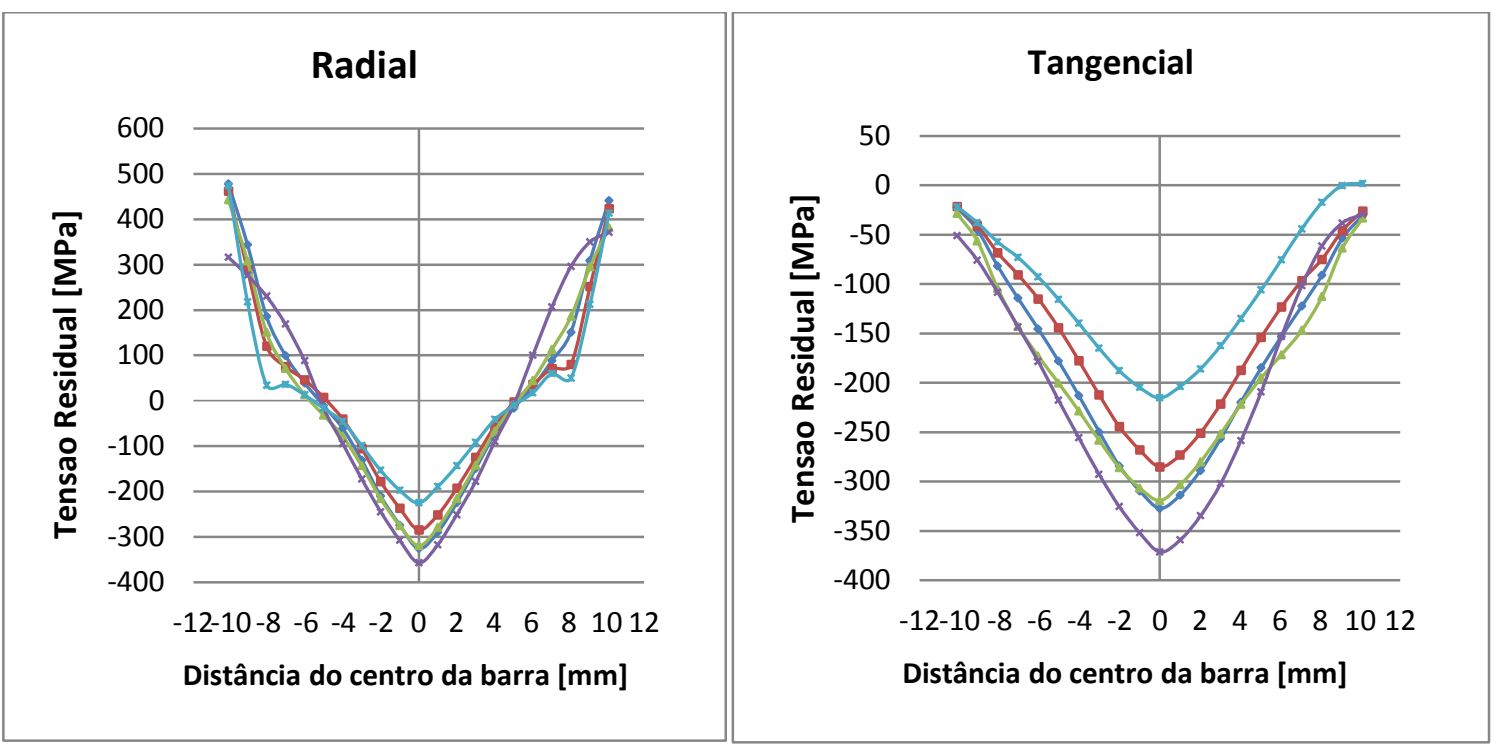

Na legenda: o valor aproximados dos ângulos em graus e minutos.

Figura 5. Gráficos das tensões residuais do estudo dos ângulos nos perfis axial, radial e tangencial.

\subsection{Região de Calibração}

As simulações para análise da influência da região de calibração foram feitas no Deform 2D, em função do tempo computacional . Neste modelo, o processo ocorre com um eixo de simetria e o desenho da ferramenta é facilmente alterável. Foram variados os passes de 1 a 5 , em que cada unidade a mais significa um aumento de $5 \mathrm{~mm}$ na região, isto pode ser verificado na Figura 6 em que aparece em escala de cores o resultado das tensões residuais.

* Contribuição técnica ao $69^{\circ}$ Congresso Anual da ABM - Internacional e ao 14ํㅡㄹ ENEMET - Encontro Nacional de Estudantes de Engenharia Metalúrgica, de Materiais e de Minas, 21 a 25 de julho de 2014, São Paulo, SP, Brasil. 


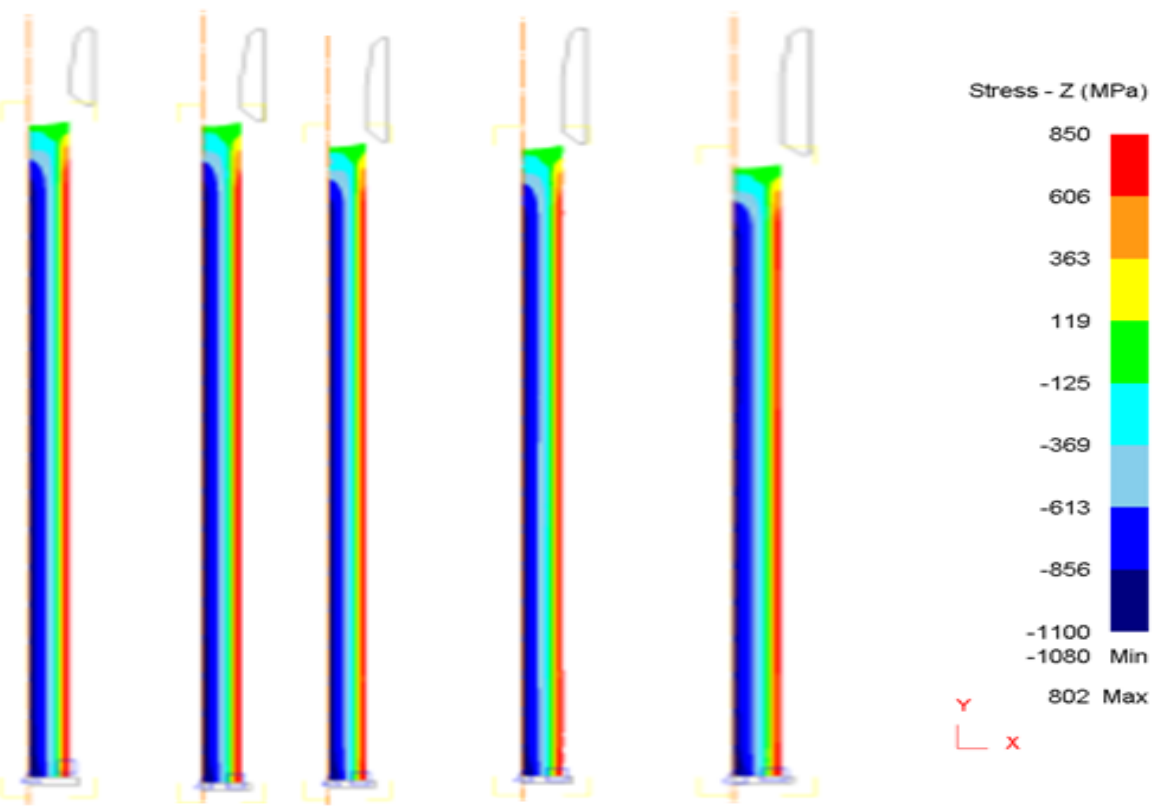

As barras apresentadas na Figura 6 estão na ordem crescente em relação ao aumento dos passes de 1 a 5 .

Figura 6. Perfil das tensões residuais para avaliação da influência dos passes.

O gráfico está representado na Figura 7 em que se tornam visíveis as diferenças dos valores de tensões obtidos nas simulações.
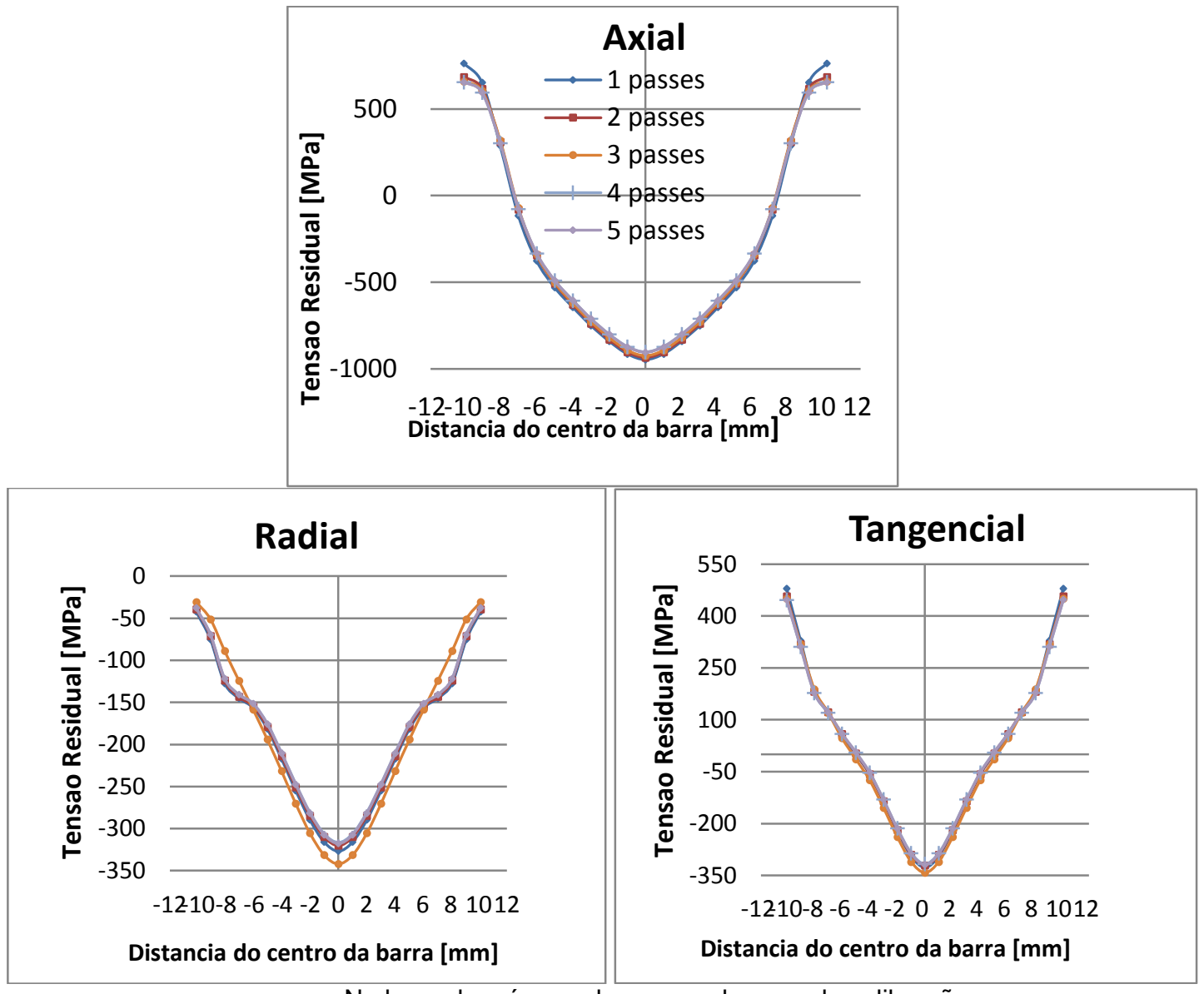

Na legenda: número de passes da zona de calibração.

Figura 7. Gráficos das tensões residuais do estudo dos passes nos perfis axial, radial e tangencial.

* Contribuição técnica ao 69 Congresso Anual da ABM - Internacional e ao 14ํㅡㄹ ENEMET - Encontro Nacional de Estudantes de Engenharia Metalúrgica, de Materiais e de Minas, 21 a 25 de julho de 2014, São Paulo, SP, Brasil. 


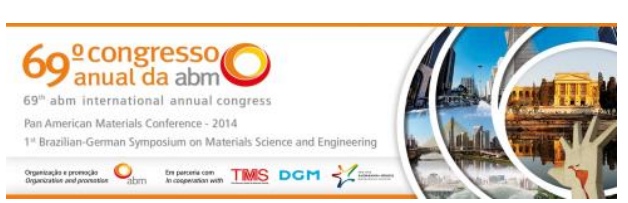

Nas Tabelas 8, 9 e 10 estão inseridos os valores das tensões na superfície e no centro das barras, em que tendem à diminuição dos valores nas superfícies e aumento no centro conforme a ordem do número de passes.

Tabela 8. Tensões na direção radial Axial

\begin{tabular}{cccc}
\hline Angulo & Superfície & Centro & Superfície \\
\hline 1 & 764 & -946 & 764 \\
\hline 2 & 685 & -934 & 685 \\
\hline 3 & 660 & -923 & 660 \\
\hline 4 & 655 & -902 & 655 \\
\hline 5 & 655 & -904 & 655 \\
\hline
\end{tabular}

Tabela 9. Tensões na direção radial

\begin{tabular}{cccc}
\hline Ângulo & Superfície & Centro & Superfície \\
\hline 1 & -42 & -327 & -42 \\
\hline 2 & -39 & -320 & -39 \\
\hline 3 & -31 & -342 & -31 \\
\hline 4 & -38 & -316 & -38 \\
\hline 5 & -38 & -318 & -38 \\
\hline
\end{tabular}

Tabela 10. Tensões na região Tangencial

\begin{tabular}{cccc}
\hline Angulo & Superfície & Centro & Superfície \\
\hline 1 & 479 & -327 & 479 \\
\hline 2 & 458 & -321 & 458 \\
\hline 3 & 451 & -342 & 451 \\
\hline 4 & 447 & -317 & 447 \\
\hline 5 & 448 & -318 & 448 \\
\hline
\end{tabular}

Nota-se que as tensões nas superfícies chegam no máximo a um decréscimo de $14 \%$ e no centro de $4 \%$ em valor modular.

\section{Conclusão}

Foram visíveis certas regularidades nas mudanças dos gráficos das tensões residuais entre os ângulos, em que podemos notar a queda do valor nas superfície das barras quanto menor for o ângulo de entrada, isto pode estar relacionado à pequena redução do diâmetro no processo referido e ao material trefilado, mas também aparece certa irregularidade no perfil das tensões quando há aumento da área de redução, podendo ser visto na tabela de forças (Tabela 3), em que apontou a maior força utilizada no processo dentre todas as simulações, mostrando que o software não o representa como em teoria.

O software também mostra que a menor força de trefilação foi com a fieira de $15^{\circ}$ os outros ângulos tiveram aumento visível, portanto resta para próximos estudos verificar qual seria o melhor modelo de geometria entre essas diferentes fieiras que possua os resultados adequados com as exigências reais no processo da trefilação. Além disso, torna-se necessário verificar o quanto essas simulações se aproximam do processo real utilizando outros parâmetros; pois, comparando a simulação da fieira de $15^{\circ} \mathrm{com}$ uma de $20^{\circ}$ com outro método de interação apresentado por Zottis [7], pode-se verificar uma diferença muito maior de aproximadamente $200 \mathrm{MPa}$, isto também é um ponto relevante na medida que o software pode variar muito os resultados mesmo que a geometrias das ferramentas sejam as mesmas. A simulação com ângulo de $10,692^{\circ}$ teve uma redução do valor das tensões

* Contribuição técnica ao $69^{\circ}$ Congresso Anual da ABM - Internacional e ao 14ํㅡㄹ ENEMET - Encontro Nacional de Estudantes de Engenharia Metalúrgica, de Materiais e de Minas, 21 a 25 de julho de 2014, São Paulo, SP, Brasil. 


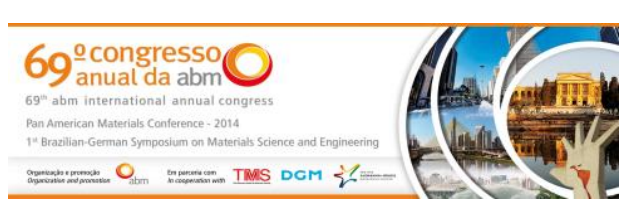

superficiais de aproximadamente $44 \%$ em relação a de $15^{\circ}$ graus e no centro o valor das tensões aumentou em aprox. 5\%. Esses resultados mostram a importância da diminuição do ângulo de trabalho neste caso, sendo que talvez seja possível corrigir ainda mais a diferença das tensões no centro das barras com o aumento dos passes da ferramenta, visto que a força de trefilação aumentaria no máximo em $4 \%$ com o acréscimo de 1 passe.

Comparando a fieira de ângulo de entrada de $14,358^{\circ} \mathrm{com}$ a de $15^{\circ}$, nota-se mais uma vez maior regularidade e diminuição das tensões residuais na superfície das peças, confirmando que para o valor de redução do diâmetro das ferramentas e atrito em questão, as respostas são satisfatórias para os ângulos menores do que $15^{\circ}$.

Ainda que tenham sido apresentadas boas opções de ferramenta, deve-se verificar o quanto isto significa para a melhoria de uma produção. É nesse contexto que a simulação computacional como método permite ainda que sejam realizadas, após uma melhor precisão do entendimento dos resultados, uma combinação entre todos esses diferentes parâmetros, junto a outros tipos de experimentos. Por isso, em próximos estudos, deveriam ser verificados outros métodos de simulação para as mesmas geometrias de ferramenta para pontuar a precisão do software de modo que possam estar suficientemente próximos de valores experimentais ou até mesmo dos cálculos em literaturas.

\section{Agradecimentos}

Agradecemos à Capes, o CNPq e a FINEP pelo apoio financeiro, ao programa Bragecrim (Brazilian German Collaborative Research Initiative in Manufacturing Technology), que possibilitou que o desenvolvimento e continuação desta pesquisa, e a FAPERGS, pelo financiamento da bolsa de iniciação científica.

\section{REFERÊNCIAS}

1 Wright RN. Wire Technology Process Engineering and Metalurgy.

2 Martins,P., Extrusão e Trefilagem. Material didático, Instituto superior técnico. Universidade técnica de lisboa. Portugal, 2012.

3 Soares CAT. Análise das Tensões Residuais no Processo de Trefilacão Considerando os Efeitos de Anisotropia. Dissertação de Mestrado - Programa de Pós Graduação em Engenharia de Minas, Metalúrgica e de Materiais - PPGE3M - UFRGS. Porto Alegre, 2012.

4 Atienza JM. Tensiones Residuales En Alambres de Acero Trefilados. Tesis Doctoral. Universidad Politécnica de Madrid, 2001.

5 Rubio EM, Camacho AM, Sevilla L, Sebastian MA.Calculation of the Forward Tension in Drawing Processes. Journal of Materials Processing Technology, 2005; 162-163: 551-557.

6 Tekkaya AE. State-of-the-Art of Simulation of Sheet Metal Forming.Journal of Material Processing Technology , 2000; 103: 14-22.

7 Zottis J.; Modelagem e Análise Numérica na Precisão de Distorções no Processo de Trefilação de Barras de Aço. Dissertação de Mestrado. Porto Alegre; 2013.

8 Wang Z, Gong B. Residual Stress in the Forming of Materials. In Handbook of Residual Stress and Deformation of Steel. 2002, p. 141-49.

9 Souza, TF. Simulações Computacionais para Análise e Minimização das Tensões Residuais no Processo de Trefilação. Dissertação de Mestrado - Programa de Pós Graduação em Engenharis de Minas, Metalúrgica e de Materiais - PPGE3M - UFRGS. Porto Alegre, 2011.

\footnotetext{
* Contribuição técnica ao $69^{\circ}$ Congresso Anual da ABM - Internacional e ao 14ํㅡㄹ ENEMET - Encontro Nacional de Estudantes de Engenharia Metalúrgica, de Materiais e de Minas, 21 a 25 de julho de 2014, São Paulo, SP, Brasil.
} 